\title{
Introduction to the Philosophy of Science
}

\author{
Antoni Diller
}

1 December 2011

\section{Introduction}

The following are some of the questions that philosophers of science study:

- How do theories come about?

- What makes one theory better than another?

- Why do scientists reject some theories?

I will first look at the traditional answers given to these questions and give some reasons why these traditional answers are mistaken, then I will look at Popper's influential work on these same problems.

\section{The Traditional View of the Scientific Method}

\subsection{Introduction}

The traditional view of the scientific method sees the formation and justification of theories occurring in the following order:

(1) Observe

(2) Theorise

(3) Verify

Apparently, this view of the scientific method will still be held by some in the 24th century AD as Dr Crusher espouses it in the Star Trek: The Next Generation episode "Home Soil"! I will now briefly consider the three steps of the traditional understanding of the scientific method.

\subsection{Observation}

Observation is not as straightforward as it may seem. Popper makes the following point in his book Conjectures and Refutations [8, p. 46], '[The] belief that we can start with pure observations alone, without anything in the nature of a theory, is absurd'. He elaborates this as follows: 
Twenty-five years ago I tried to bring home the same point to a group of physics students in Vienna by beginning a lecture with the following instructions: "Take pencil and paper; carefully observe, and write down what you have observed!" They asked, of course, what I wanted them to observe. Clearly the instruction, "Observe!" is absurd ... Observation is always selective. It needs a chosen object, a definite task, an interest, a point of view, a problem. And its description presupposes a descriptive language, with property words; it presupposes similarity and classification, which in its turn presupposes interests, points of view, and problems.

Einstein makes a similar point (quoted by Brush [3, p. 1167]):

It may be heuristically useful to keep in mind what one has observed. But on principle it is quite wrong to try grounding a theory on observable quantities alone. In reality the opposite happens. It is the theory which determines what we can observe .... .

\subsection{Theory Formation}

Many people see induction as being the logical procedure which produces universal theories out of particular observation reports. Imagine that you are interested in the colour of swans. You begin by observing a large number of swans and you notice that they are all white. Using an inductive inference you conclude that all swans are white. The inductive inference would look something like this:

$$
\frac{\mathrm{Swan}_{1} \text { is white } \mathrm{Swan}_{2} \text { is white } \ldots \mathrm{Swan}_{n} \text { is white }}{\text { Every swan is white. }}
$$

The following points can be made about inductive inferences:

- Inductive inferences do not establish their conclusions.

- Positive instances are easy to find and have little value.

- Trying to find a counter-example or falsifying instance and failing is more valuable than any number of confirming or supporting instances.

- Induction is useful as a heuristic.

I will give two examples of the usefulness of induction as a heuristic. The first is known as the Pessimistic Induction:

Newton's theory is false Kepler's theory is false ... Copernicus's theory is false All scientific theories are false.

The second example of the usefulness of induction as a heuristic is provided by the movement of the planets around the Sun:

Mars moves an an ellipse around the Sun

Venus moves an an ellipse around the Sun

Jupiter moves an an ellipse around the Sun

All planets move in ellipses around the Sun. 


\subsection{Verification}

In the traditional understanding of the scientific method the probability of the truth of a theory increases with every instance that confirms that theory. The more white swans you see the greater the probability that all swans are white. There are many problems with such an account of confirmation. I will mention just one. A universal statement like 'Every swan is white' is said to be confirmed by the observation of a white swan. The statement 'Every swan is white', however, is equivalent to the statement 'Every non-white thing is not a swan' and this is confirmed by the observation of every nonwhite non-swan. This, however, is highly counter-intuitive. We feel the observation of a red car should not increase the probability of the truth of the statement 'Every swan is white'.

\section{Popper's Tetradic Problem-solving Schema}

\subsection{Introduction}

Popper's account of how science progresses is summed up in the following tetradic problem-solving schema that occurs frequently in his writings. (It is found, for example, in Objective Knowledge on pp. 119, 121, 126, 144, 164, 168, 243, 287 and 297.)

$$
P_{1} \longrightarrow T T \longrightarrow E E \longrightarrow P_{2} \text {. }
$$

The various symbols in this schema have the following meanings:

$P_{1}$ is the initial problem.

$T T$ is a tentative theory put forward to solve $P_{1}$.

$E E$ is the process of error elimination in which $T T$ is thoroughly criticised.

$P_{2}$ is a problem thrown up by the error-elimination process.

In Popper's schema $P_{1}$ can be either a practical problem or a theoretical problem. He gives the following example when it is a practical problem (from Knowledge and the Body-mind Problem (1994), p. 11):

Henry Ford's original problem was: how can we provide transport for the vast spaces of the United States? This was his $P_{1}$. He proposed the theory: by building a cheap motor car. This led through various trials and errors to a new problem: how can we provide the roads and parking places needed for your cars? The original problem $P_{1}$ was the problem of transport. The new problem $P_{2}$ is the traffic problem - a problem of frustration.

I will now briefly consider the various components that occur in Popper's schema. 


\subsection{Problems}

Popper stressed the importance of problems to science. For example, in Realism and the Aim of Science (1983), p. 8, he writes:

I think that there is only one way to science - or to philosophy, for that matter: to meet a problem, to see its beauty and fall in love with it; to get married to it, and to live with it happily, till death do ye part-unless you should meet another and even more fascinating problem, or unless, indeed, you should obtain a solution. But even if you do obtain a solution, you may then discover, to your delight, the existence of a whole family of enchanting though perhaps difficult problem children for whose welfare you may work, with a purpose, to the end of your days.

\subsection{Theory Creation}

There is no rational method of coming up with new ideas. Devising theories is an irrational process. That does not make science irrational. Philosophers of science distinguish between a context of discovery and a context of criticism. What makes science rational is the thorough criticism that theories undergo before they are admitted into the fabric of knowledge. Here are some examples of how various ideas were obtained:

- dream (Kekulé)

- alchemy and Sun-worship (Copernicus)

- seance (photocopying)

- LSD (Francis Crick)

One of the reason why theory creation is irrational is that newer scientific theories are usually inconsistent with earlier ones that solve similar problems. The diagram shown in Fig. 1 shows how knowledge is modified. Note especially that each pair of adjacent theories is mutually inconsistent:

Aristotle-Galileo One of the many differences between Aristotle's mechanics and Galileo's is that, according to Aristotle, if you drop two objects from a great height, the heavier one will hit the ground more quickly than the lighter one. Galileo famously dropped two objects of unequal weight from the top of the Leaning Tower of Pisa and they hit the ground at the same time. His mechanics could account for this phenomenon.

Galileo-Newton (1) According to Galileo's mechanics a projectile fired from the surface of the Earth moves in a parabola, but according to Newtonian mechanics it moves in an ellipse. (2) According to Galileo's mechanics a free-falling body (for example, dropped from a high object) has constant acceleration, but according to Newtonian mechanics its acceleration increases slightly as it moves closer to the centre of the Earth. 


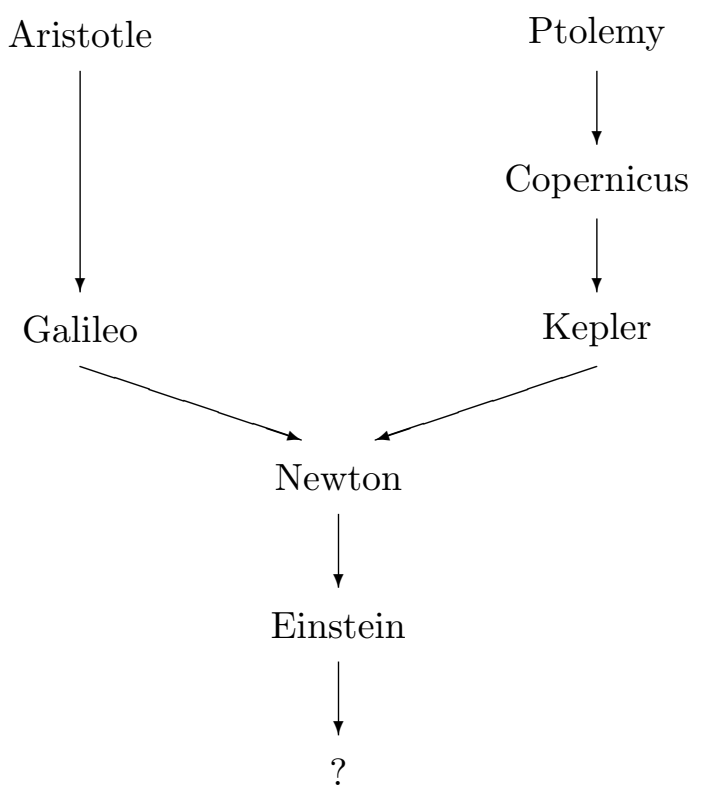

Figure 1: Illustrating how knowledge is modified.

Ptolemy-Copernicus For Ptolemy the planets and the Sun moved in circles around the Earth, whereas for Copernicus, the planets, including the Earth, moved in circles around the Sun.

Copernicus-Kepler According to Copernicus, the planets moved in circles around the Sun, whereas, according to Kepler, they moved around the Sun in ellipses.

Kepler-Newton In framing his laws of planetary motion Kepler ignores the mutual attraction that exists between heavenly bodies. Thus, according to Kepler's third law $a^{3} / T^{2}=k$, where $a$ is the mean distance between two bodies with mass $m_{0}$ and $m_{1}, T$ is the time it takes for one complete revolution of one about the other and $k$ is a constant. For Newton, however, $a^{3} / T^{2}=m_{0}+m_{1}$.

Newton-Einstein There are many differences between Newtonian and Einsteinian mechanics. In Einstein's theory of relativity nothing can travel faster than the speed of light, whereas there is no maximum velocity in Newtonian mechanics. In Einsteinian mechanics two events are only simultaneous relative to a frame of reference, whereas in Newtonian mechanics events are simultaneous or nonsimultaneous absolutely.

Reflecting on the fact that later scientific theories contradict earlier ones, in his book Objective Knowledge [9, p. 266], Popper gives the following advice: 
Whenever a theory appears to you as the only possible one, take this as a sign that you have neither understood the theory nor the problem which it was intended to solve.

Feyerabend goes further and enunciates his notorious Principle of Proliferation (quoted from Preston, Feyerabend (1997), p. 138):

Invent, and elaborate, theories which are inconsistent with the accepted point of view, even if the latter should happen to be highly confirmed and generally accepted.

\subsection{Error Elimination}

Theories can be criticised in very many different ways. Here I will give a few examples of how various different sorts of theory can be criticised. The following suggestions about how to criticise a theory are based on the ideas of Bartley [1, p. 127] and Laudan [6, ch. 2]:

- Is this theory consistent?

- Is this theory empirical?

- Is this theory better than its rivals?

- Is this theory consistent with observed facts?

- What problem is this theory intended to solve?

- Does this theory successfully solve the problem it was put forward to solve?

- Does it, maybe, solve some other problem?

- Is this theory in conflict with some other scientific theory that is well established?

- Is this theory in conflict with the methodology of its parent discipline?

- Is this theory in conflict with some element of the dominant cultural worldview?

\subsection{Elaboration of Popper's Schema}

Popper proposed several variants of his tetradic problem-solving schema. Fig. 2 shows a variant (based on the diagram found in Objective Knowledge, p. 243) in which $n$ theories are put forward to solve a single problem $P_{1}$. They are then criticised and evaluated as a group and this process of error-elimination results in a new problem $P_{2}$. Fig. 3 is a variant in which again there are $n$ solutions to $P_{1}$, but the process of error-elimination is different for each theory. Furthermore, each critical discussion of a conjectural theory results in a different novel problem. (Fig. 3 is based on diagrams found in Objective Knowledge, p. 287, and Knowledge and the Body-mind Problem, p. 12.) Yet another possibility is shown in Fig. 4. This is similar to Popper's original schema $P_{1} \longrightarrow T T \longrightarrow E E \longrightarrow P_{2}$, except that $n$ new problems are thrown up by the error-elimination process. 


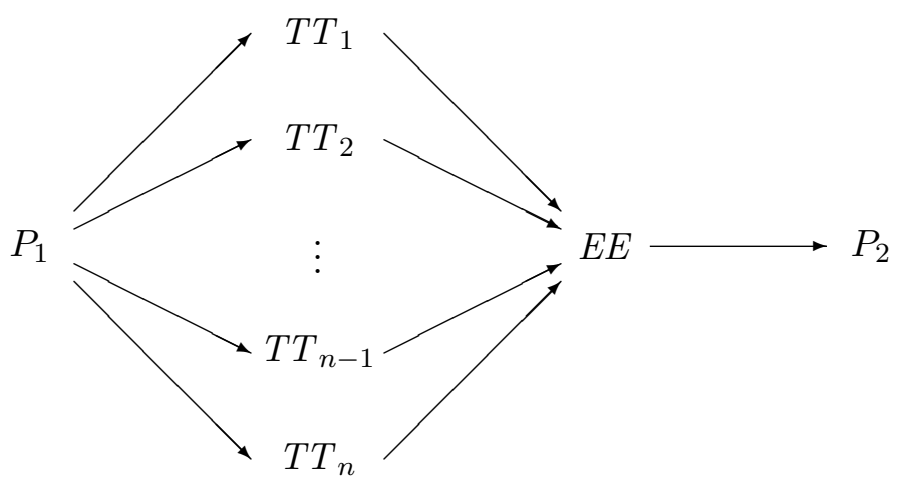

Figure 2: A variant of Popper's schema.

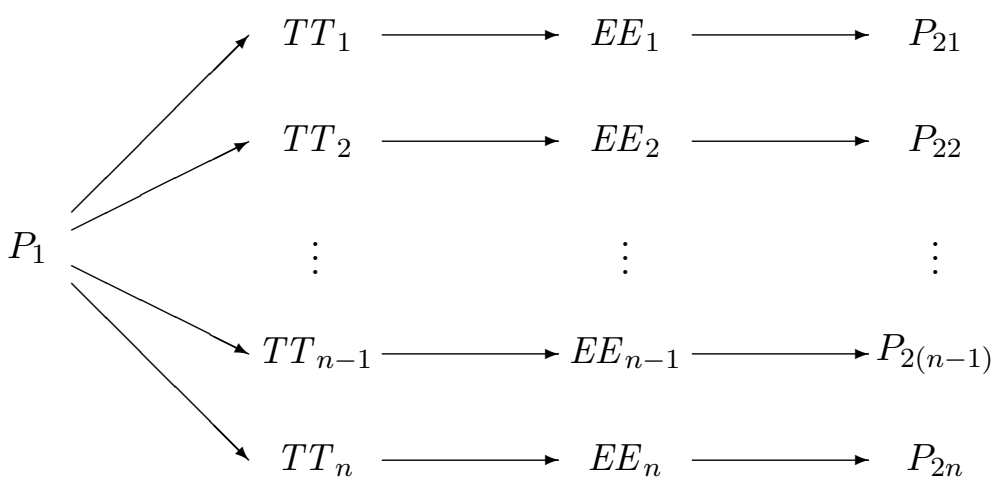

Figure 3: A variant of Popper's schema.

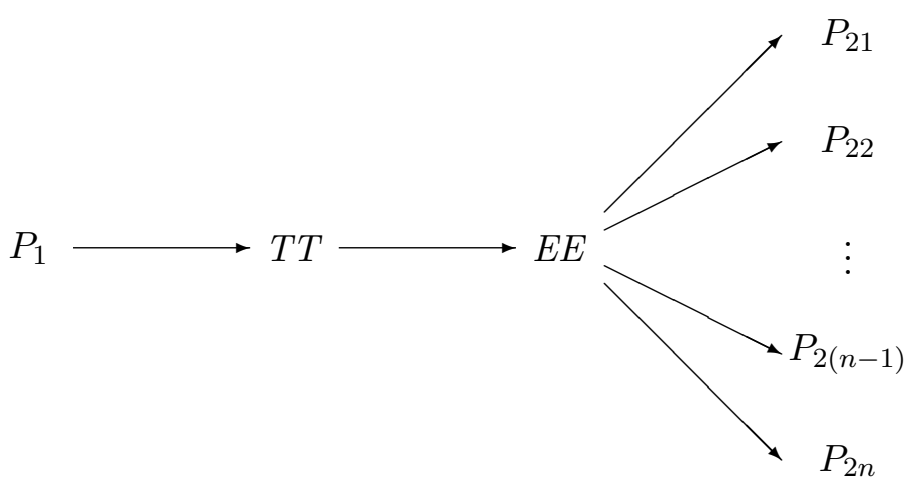

Figure 4: A variant of Popper's schema. 


\section{Examples of Epistemological Problems}

The traditional account of the scientific method that I sketched above is thoroughly justificationist in its outlook, whereas the Popperian account of how science progresses is anti-justificationist. In Table $1 \mathrm{I}$ list some examples of justificationist problems and some examples of anti-justificationist ones.

\begin{tabular}{|c|c|}
\hline justificationist & anti-justificationist \\
\hline What do we know? & How does our knowledge grow? \\
\hline What can we know? & How can we get rid of false theories? \\
\hline $\begin{array}{l}\text { How can we justify our theories or } \\
\text { beliefs? }\end{array}$ & $\begin{array}{l}\text { What are good ways of criticising } \\
\text { theories }\end{array}$ \\
\hline What does a justification consist of? & $\begin{array}{l}\text { How can we decide which out of a } \\
\text { group of competing theories is the } \\
\text { best? }\end{array}$ \\
\hline $\begin{array}{l}\text { Is it possible to justify our theories } \\
\text { rationally? }\end{array}$ & \\
\hline $\begin{array}{l}\text { Can we justify our belief in the ex- } \\
\text { istence of other minds and how? }\end{array}$ & $\begin{array}{l}\text { Is the theory that other minds exist } \\
\text { better or worse than the theory that } \\
\text { they do not? }\end{array}$ \\
\hline
\end{tabular}

Table 1: Examples of epistemological problems..

\section{Explanation, Prediction and Falsification}

A very common pattern of explanation is known as the covering law model or the deductive-nomological (DN) model. The pattern is as follows:

$$
\frac{U \quad I}{E,}
$$

where $E$ is a statement of the fact being explained, $U$ is a universal scientific law and $I$ is a statement of the initial conditions. An example of an explanation is the following:

universal scientific law Whenever a thread is loaded with a weight exceeding that which characterizes the tensile strength of the thread, then it will break.

initial conditions The weight characteristic for this thread is 1lb. The weight put on this thread was $2 \mathrm{lbs}$.

statement of the fact being explained (That is why) this thread broke.

The DN-model can also be used to make predictions of what should happen when certain initial conditions obtain. If the prediction turns out to be false, assuming that the initial conditions actually did obtain, then the universal scientific law has been 
falsified. Many scientists are not happy when their pet theories are falsified, so they sometimes try to immunise their theories from falsification and explain away putative falsifications. This does nothing to advance science. Unfortunately, it does happen in AI and Cognitive Science. Dreyfus in his book What Computers Still Can't Do (1992), describes a program written by Allen Newell and H.A.Simon which they called the General Problem Solver or GPS. They thought that this replicated human thought and they dismissed evidence to the contrary. Dreyfus gives several examples. Here is one (from p. 112):

Comparing the machine print-out of the steps of a GPS solution with the transcript of the verbal report of a human being solving the same problem reveals steps in the machine trace (explicit searching) which do not appear in the subject's protocol. And Simon asks us to accept the methodologically dubious explanation of the missing steps in the human protocol that "many things concerning the task surely occurred without the subject's commenting on them (or being aware of them)" and the even more arbitrary assumption that these further operations were of the same elementary sort as those verbalized.

(The quotation within this quotation is from p. 288 of Newell and Simon's "GPS: A Program That Simulates Human Thought" [7]. Note that a "protocol is a verbal report of a subject in the process of solving a problem' [4, p. 318, n. 8].) Dreyfus also gives the following example of how Newell and Simon dismiss falsifications of their theory that GPS thinks like a human (p. 113):

In one of Simon's experiments, subjects were given problems in formal logic and a list of rules for transforming symbolic expressions and asked to verbalized their attempt to solve the problems. The details of the rules are not important; what is important is that at a point in the protocol the subject notes that he applies the rule $(A \cdot B \rightarrow A)$ and the rule $(A \cdot B \rightarrow B)$, to the conjunction $(-R \vee-P) \cdot(R \vee Q)$. Newell and Simon comment:

The subject handled both forms of rule 8 together, at least as far as his comment is concerned. GPS, on the other hand, took a separate cycle of considerations for each form. Possibly the subject followed the program covertly and simply reported the two results together.

(The quotation within this quotation is from p. 289 of Newell and Simon's "GPS: A Program That Simulates Human Thought" [7].) It is much better to give up a falsified theory and look for a better one than try to save the falsified theory by explaining away the falsifications.

\section{The Knowledge Filter}

It is not easy to accept things that are inconsistent with our current beliefs. Some philosophers of science call this the knowledge filter (see Fig. 5). We have a tendency 


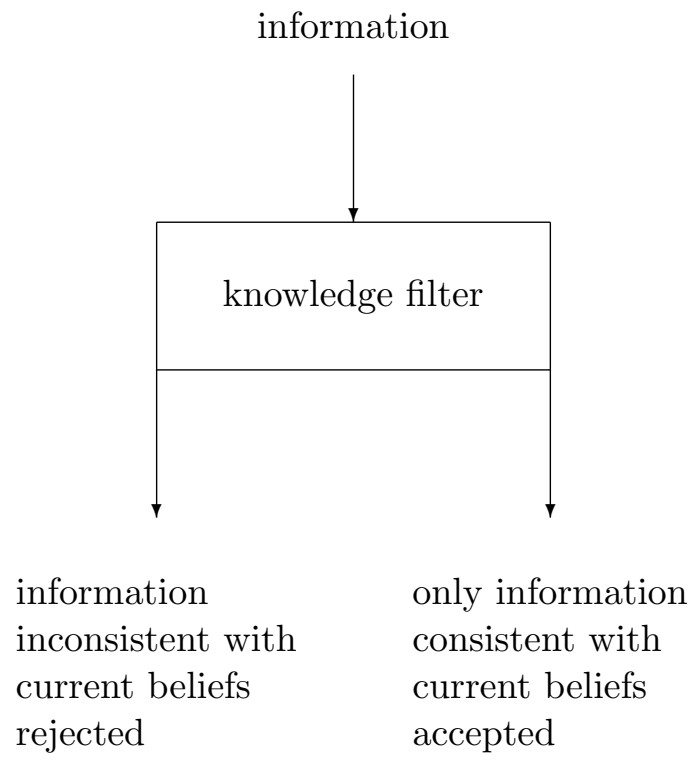

Figure 5: The knowledge filter.

to disregard information that would cause us to re-evaluate our most cherished beliefs. Charles Darwin was implicitly aware of knowledge filtration and worked against it as he wrote (in his Autobiography, p. 123):

I had during many years followed a golden rule, namely, that whenever a published fact, a new observation or thought came across me, which was opposed to my general results, to make a memorandum of it without fail and at once; for I had found by experience that such facts and thoughts were far more apt to escape from the memory than favourable ones. Owing to this habit, very few objections were raised against my views which I had not at least noticed and attempted to answer.

In his very influential book, The Structure of Scientific Revolutions [5, pp. 62-63], Kuhn describes a fascinating psychological experiment which well illustrates knowledge filtration [2]:

In a psychological experiment that deserves to be far better known outside the trade, Bruner and Postman asked experimental subjects to identify on short and controlled exposure a series of playing cards. Many of the cards were normal, but some were made anomalous, e.g., a red six of spades and a black four of hearts. Each experimental run was constituted by the display of a single card to a single subject in a series of gradually increased exposures. After each exposure the subject was asked what he had seen, and the run was terminated by two successive correct identifications. 
Even on the shortest exposures many subjects identified most of the cards, and after a small increase all the subjects identified them all. For the normal cards these identifications were usually correct, but the anomalous cards were almost always identified, without apparent hesitation or puzzlement, as normal. The black four of hearts might, for example, be identified as the four of either spades or hearts. Without any awareness of trouble, it was immediately fitted to one of the conceptual categories prepared by prior experience. One would not even like to say that the subjects had seen something different from what they identified.

\section{References}

[1] William Warren Bartley, III. The Retreat to Commitment. Open Court Publishing Company, La Salle and London, second edition, 1984.

[2] J. S. Bruner and Leo Postman. On the perception of incongruity: A paradigm. Journal of Personality, XVIII:206-223, 1949.

[3] S. G. Brush. Should the history of science be rated X? Science, 183:1164-1172, 1974

[4] Hubert L. Dreyfus. What Computers Still Can't Do: A Critique of Artificial Reason. The MIT Press, Cambridge (Massachusetts), 1992.

[5] Thomas S. Kuhn. The Structure of Scientific Revolutions, volume 2.2 of International Encyclopedia of Unified Science, editor-in-chief: Otto Neurath. University of Chicago Press, London, second edition, 1970.

[6] Larry Laudan. Progress and its Problems: Towards a Theory of Scientific Growth. Routledge \& Kegan Paul, London, 1977.

[7] Allen Newell and H. A. Simon. GPS: A program that simulates human thought. In Edward A. Feigenbaum and Julian Feldman, editors, Computers and Thought. McGraw-Hill, New York, 1963.

[8] Karl Raimund Popper. Conjectures and Refutations: The Growth of Scientific Knowledge. Routledge and Kegan Paul, London, fifth edition, 1974. Originally published in 1963.

[9] Karl Raimund Popper. Objective Knowledge: An Evolutionary Approach. Oxford University Press, London, 1975. Originally published in 1972.

[10] Karl Raimund Popper. Realism and the Aim of Science. Routledge, London and New York, 1983. From the Postscript to the Logic of Scientific Discovery. Edited by W.W. Bartley, III.

[11] Karl Raimund Popper. Knowledge and the Body-mind Problem: In Defence of Interaction. Routledge, London, 1994. Edited by M. A. Notturno. 
[12] John Preston. Feyerabend: Philosophy, Science and Society. Polity Press, Cambridge, 1997. 